\title{
Association of the polymorphisms P53 gene with juvenile idiopathic arthritis in children Russian Fedration
}

\author{
Alexey N Kozhevnikov ${ }^{1 *}$, Michael V Moskalenko ${ }^{3}$, Nina A Pozdeeva ${ }^{2}$, Margarita F Dubko ${ }^{1}$, Valentina I Larionova ${ }^{1}$, \\ Gennagy A Novik ${ }^{1}$
}

From 18th Pediatric Rheumatology European Society (PReS) Congress

Bruges, Belgium. 14-18 September 2011

\section{Background}

Juvenile idiopathic arthritis - is a chronic systemic autoimmune disease that is a characterized by articular lesion with synovial hyperplasia and cellular infiltration. Arg72Pro (4ex) and ins/del16bp (3in) polymorphisms are associated with affects the functional activity of the p53 protein. (P.Dumont et al, 2003)

\section{Aim}

The purpose of our study is estimate course and outcomes of juvenile idiopathic arthritis of the children with various genotypes of $p 53$.

\section{Methods}

We examined 58 children with juvenile idiopathic arthritis. Clinical, serological and $\mathrm{x}$-ray manifestations were analyzed in children and correlated with the genotypes. For detection erosion bone process we used ultrasound, x-ray, MRI and diagnostic arthroscopy with synovial biopsy. We investigated (PCR-RFLP) the status of p53 gene this children with juvenile idiopathic arthritis and 100 healthy children living in Russian Federation.

\section{Results}

Genotypes distributions of Arg72Pro and ins/del16bp polymorphisms did not differ significantly $(\mathrm{p}>0,05)$ between JIA patients and controls. Children with mild form oligo-, polyarthritis JIA achieved remission had significantly higher percentage genotype $\mathrm{Arg} / \mathrm{Arg}+\mathrm{del} / \mathrm{del}$ compared children with severe oligo, polyarthritis duration more 5 years $(89,7$ vs $23,8 \%, p<0,01)$. Young girls with severe oligoarthirits, ANA-positive and erosion joint process had a significantly higher percentage of genotype $\mathrm{Arg} / \mathrm{Pro}+\mathrm{ins} / \mathrm{del}$ compared children with mild form oligoarthritis, ANA-negative $(87,5$ vs $9 \%, \mathrm{p}<0,01)$. Girls with severe polyarthritis DAS44 4.0 \pm 1.1 had significant high percentage genotype Arg/Pro+ins/del compared children with mild form polyarthritis DAS44 $2.4 \pm 0.9$ (67 vs $9 \%, \mathrm{p}<0,01)$.

\section{Conclusion}

Girls with genotype Arg/Pro+ins/delp53 had more severe and aggressive form oligo-polyarthritis manifested by erosion process.

\section{Author details}

'Saint-Petersburg Pediatric Medical Academy, Saint-Petersburg, Russian Federation. ${ }^{2}$ Saint-Petersburg Research Pediatric Orthopedic Institute n. a. G.I. Turner, Saint-Petersburg, Russian Federation. ${ }^{3}$ Saint-Petersburg State University, Department Genetics \& Breeding. Saint-Petersburg, Russian Federation.

Published: 14 September 2011 